\title{
Designing laser resonators with a specified output
}

\author{
P.A. BÉLANGER and C. PARÉ \\ Université Laval, Québec, Canada
}

\section{INTRODUCTION}

Optical laser resonators generally consist of open cavities formed with two spherical mirrors enclosing gain and modulation elements. The analysis of the spherical resonator is now standard [1-2] and leads to a classification of the resonator as "stable" or "unstable" cavities. To be of good quality, a laser beam must oscillate in only one transverse mode which should cover a large fraction of the gain section of the amplifying medium. Owing to its geometrical magnification factor, the unstable resonator offers the advantage of providing a large mode volume as well as a high discrimination against the perturbing higher-order transverse modes. However, the highly diffracted output of such resonators is a severe drawback for some applications. On the other hand, the output beam of the stable resonators generally presents an ideal propagation behaviour. The stable cavity has a low-loss confined mode but its beam size is rather small for typical experimental conditions where the stability against misalignment is always important. Moreover, this resonator presents a poor modal selectivity since the higher-order modes are also confined and nearly lossless.

In the past, workers have tried to combine the advantages of both types of resonator in the same cavity. Most of these efforts consisted in reducing the hardedged diffraction of the unstable resonator by, for example, changing locally the mirror curvature near the edges or by introducing phase shifts near the edges or on the Fresnel zones [1]. Over the past few years, it has been widely recognized that the hard-edged diffraction in an unstable resonator can be best controlled by the use of a graded-reflectivity mirror (GRM) [1]. This confines the beam as in a stable cavity but the beam waist can be adjusted to the size of the gain medium.

For the important class of medium- and low-gain lasers, a different solution for increasing the beam size still has to be demonstrated. If properly designed, the optical resonator formed with an aspherical mirror and a plane output coupler seems to us to be able to meet these specifications. In the past, only a few analyses (that we know of) of this type of resonator have been published [2,3]. The absence of exhaustive conclusions on the properties of aspherical resonators is largely due to the fact that such an investigation would imply numerical solution of coupled integral equations with a large number of parameters needed for specifying a sufficiently wide class of aspherical mirrors and the resonator dimensions. 


\section{2. "Custom phase-conjugate resonators"}

More recently, we have proposed [4,5] a wide class of aspherical resonators which have the property of yielding a fundamental mode of prescribed shape and of the stable type. Our ultimate goal is a systematic specification of an aspherical resonator with (i) a predetermined fundamental mode of the stable type (low loss), and (ii) highly discrimated higher-order transverse modes. The class of aspherical mirror resonator that we have recently introduced will be referred to here as "custom phase-conjugate resonator" because the basic procedure for the design of the aspherical mirror starts with the specification by the users, of the intensity distribution on the output coupler. In this approach, the desired fundamental mode distribution is first propagated up to the mirror position; to limit the losses of this particular mode, the mirror is then made to conjugate this propagated field, i.e., that the mirror profile is chosen to correspond to the wave front of this mode at this position. In the limit of large aperture, it has been shown [5] that the desired mode is indeed a solution of the integral equation. A numerical analysis is still necessary, however, if one wants to determine the higher-order modes and, hence, possibly improve the modal selectivity. Our recent analysis of resonators with a superGaussian intensity ouput [6] indicates that this is possible for a broad band of resonator parameters; this has also been recently confirmed in the laboratory [7]. Referring to Figure 1 , two mirrors, $\mathrm{M}_{2}$ and $\mathrm{M}_{6}$, were designed using this inverse propagation method and were ground out of copper with diamond turning machining. The super-gaussian amplitude profiles specified on the plane coupler are

$$
M_{2} \rightarrow \exp \left[-(r / 2.6)^{2}\right] \text { and } M_{6} \rightarrow \exp \left[-(r / 3.3)^{6}\right]
$$

where $\mathbf{r}$ is in $\mathrm{mm}$. The mirrors were calculated to match the back-propagated wavefronts of these amplitude distributions, assuming a plane wavefront on the coupler. The first mirror is of course spherical and serves as a basis of comparison. The profile $M_{6}$ compared with that of the spherical mirror $M_{2}$ is shown in figure 1. The beamwidth parameters of $2.6 \mathrm{~mm}$ and $3.3 \mathrm{~mm}$ were chosen so that the Rayleigh range for each of these output beams corresponds to the cavity length of the resonator, i.e. $Z_{R}=L=2 \mathrm{~m}$. (The Rayleigh range is defined here according to the second-moments theory [8] ). The geometrical parameter: $G=\left(1+L^{2} / Z_{R}^{2}\right)^{-1}$ is thus 0.5 for the two resonators. The conventional one gives a monomode output power of 4.3 watts while the super-Gaussian resonator yields $25 \%$ more power for monomode operation. The complete details of the experiment will be found in reference [9]. An effective theory predicting the behaviour of the higher order modes has also been developed [10]. From this theory, an ideal custom phase-conjugate resonator having only one transverse confined mode is predicted for an hyperbolic secant profiled mirror. For low and medium gain laser, the custom phase-conjugate resonator is a direct solution for a specified beam at the output or at any working distance for a 
particular applications.

\section{3. "Graded-reflectivity mirrors unstable resonators"}

For high power lasers the discrimination of the optical resonator must be extremely large. Only the unstable resonator with an appropriate magnification parameter $\mathrm{M}$ can yield such a discrimination. The now widely implemented use of graded-reflectivity mirrors in unstable resonators has solved the problem of hard-edge diffraction in their output beam. However the output beam profile resulting from the passage of the inside beam through the GRM mirror has natural diffraction rings. Therefore severe on-axis peaking in the output beam is detrimental in certain applications. We have very recently succeeded in applying an inverse propagation method in order to find the appropriate reflectivity profile $R(r)$ of the GRM for a specified output profile I(r). For a large Fresnel number, the self-consistent equation for the transverse mode $U(r)$ of the unstable resonator is the following secular equation:

$$
\gamma \mathfrak{i}^{2} M^{2} U(M r)=R(r) U(r)
$$

We have shown that an exact solution to this equation is

$$
R(I)=\frac{\sum_{i=1}^{\infty} R_{0}^{1} I\left(M^{1} r\right)}{\sum_{i=0}^{\infty} R_{0}^{1} I\left(M^{1} r\right)}
$$

$$
\text { where } I(I)=(1-R(I)) \quad U(I)
$$

$R_{0}$ being the on-axis reflectivity.

Several reflectivity profiles for Gaussian and super-Gaussian ouput beam profiles have been calculated. An advanced design procedure that takes into account the finite Fresnel number and the saturation of the gain medium is under development. Advances in coating technology today make possible the fabrication of precise reflectivity profile such at those required here.

\section{Conclusion}

We have shown that it is possible to design laser resonators having a specified output. The custom phase-conjugate resonator has already been proven to be a simple and direct solution to the problem for low gain laser such as gas laser. An inverse propagation method has here been derived that shows how to calculate the 
reflectivity profile of GRM unstable resonator [11]. Finally, it is important to point out that the beam can be specified also after its passage through any arbitrary optical systems. For example, it may be interesting in material processing to specify a super-Gaussian mode profile at the focus of a lens.

\section{References}

[1] A.E. Siegman, Lasers (University Science books, Mills Valley, CA, 1986), Chap. 19.

[2] Yu. A. Anan'ev, Résonateurs Optiques et Problème de Divergence du Rayonnement Laser (Editions Mir, Moscow, 1982), Chap. 2.

[3] E.F. Ishchenko and E.F. Reshetin, Opt. Spektrosk. 51, 1050 (1981) [Opt. Spectrosc. (USSR) 51, 581 (1981)].

[4] P.-A. Bélanger and C. Paré, Opt. Lett. 16, 1057 (1991).

[5] C. Paré and P.-A. Bélanger, IEEE J. Quantum Electron. QE-28, 355 (1922).

[6] C. Paré and P.-A. Bélanger, "Custom laser resonators using gradedphase mirrors: circular geometry". IEEE J. Quantum Electron. (to be published)

[7] P.-A. Bélanger, R.L. Lachance and C. Paré, Opt. Lett. 17, 739 (1992).

[8] P.-A. Bélanger, Optics Letters, vol. 16, no. 4, pp. 196-198, 1991.

[9] R. Van Neste, C. Paré, R.L. Lachance and P.-A. Bélanger, "Gradedphase mirror resonator with a super-gaussian output in a $\mathrm{CW}-\mathrm{CO}_{2}$ laser". IEEE J. Quantum Electron. (to be published).

[10] C. Paré, L. Gagnon and P.-A. Bélanger, Physical Review A, vol. 46, no. 7, pp. 4150-4160, October 1992.

[11] C. Paré and P.-A. Bélanger, "Unstable laser resonators with a specified output profile by using graded-reflectivity mirrors: Geometrical optics limit", submitted to Optics Communication, 1993.
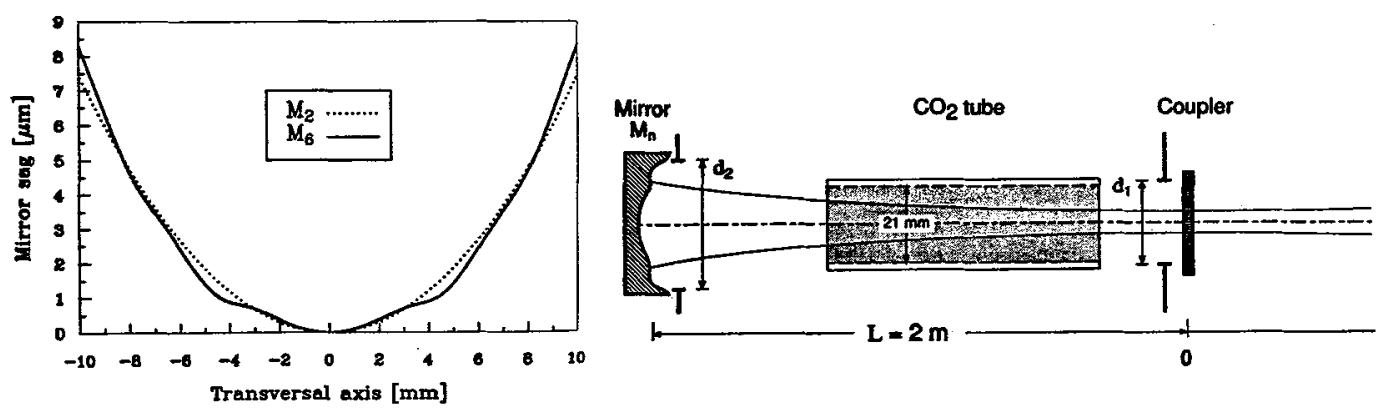

Fig. 1 - The experimental set up and the two mirror profiles $M_{2}, M_{6}$. 